\title{
Ultrafast magnetoelastic probing of surface acoustic transients
}

\author{
J. Janušonis, ${ }^{1}$ C. L. Chang, ${ }^{1}$ T. Jansma, ${ }^{1}$ A. Gatilova, ${ }^{1}$ V. S. Vlasov, ${ }^{2}$ A. M. Lomonosov,${ }^{3}$ V. V. Temnov, ${ }^{2,4}$ and R. I. Tobey ${ }^{1, *}$ \\ ${ }^{1}$ Zernike Institute for Advanced Materials, University of Groningen, Groningen, The Netherlands \\ ${ }^{2}$ IMMM CNRS 6283, Université du Maine, 72085 Le Mans cedex, France \\ ${ }^{3}$ LAUM CNRS 6613, Universite du Maine, 72085 Le Mans cedex, France \\ ${ }^{4}$ Fritz-Haber-Institut der Max-Planck-Gesellschaft, Abteilung Physikalische Chemie, Faradayweg 4-6, 14195 Berlin, Germany
}

(Received 17 January 2016; revised manuscript received 10 June 2016; published 13 July 2016)

\begin{abstract}
We generate in-plane magnetoelastic waves in nickel films using the all-optical transient grating technique. When performed on amorphous glass substrates, two dominant magnetoelastic excitations can be resonantly driven by the underlying elastic distortions, the Rayleigh surface acoustic wave and the surface skimming longitudinal wave. An applied field, oriented in the sample plane, selectively tunes the coupling between magnetic precession and one of the elastic waves, thus demonstrating selective excitation of coexisting, large-amplitude magnetoelastic waves. Analytical calculations based on the Green's function approach corroborate the generation of multiple surface acoustic transients with disparate decay dynamics.
\end{abstract}

DOI: 10.1103/PhysRevB.94.024415

\section{INTRODUCTION}

Generating elementary excitations at solid surfaces and interfaces underscores many processes in materials and enhances their use in modern technology. With the increased emphasis on new materials and enhanced functionality of existing materials, generating and detecting multiple, competing excitations at the surface provides opportunities to expanded implementation. As many of these excitations are transient in nature, the use of ultrashort optical pulses provides for the generation and real-time monitoring of their dynamics and allows for the time-domain identification of their effects on the state of the material [1].

The effects of competing excitations on material properties are exemplified in plasmonics. In research related to extraordinary transmission of light through subwavelength apertures [2-4], a long-lasting controversy exists over the nature of the responsible mechanism. It is currently understood that two competing excitations, the so-called composite diffraction evanescent waves (CDEW) and the conventional surface plasmon polaritons (SPPs), conspire to enhance transmission through apertures, while their contributions depend strongly on the experimental geometry. Alternatively, new model systems are sought in order to shed further light on extraordinary transmission effects, and acoustic analogs have been demonstrated [5].

In this article we report on the generation and selective detection of two surface acoustic waves, which are analogous to CDEW and SPP in plasmonics. The combination of femtosecond transient grating (TG) excitation with ultrafast time-resolved magneto-optical spectroscopy allows for an unambigous observation of Rayleigh surface acoustic wave (SAW) and a short-living surface skimming longitudinal wave (SSLW), both of which couple to the magnetization of the material. The differences in the acoustic properties between SAW and SSLW provides the possibility of selectively probing the individual acoustic modes via the resonant magnetoelastic excitation and represents an advantage when compared to the

\footnotetext{
*r.i.tobey@rug.nl
}

analogous plasmonic investigations, particularly in regards to the selectivity in detection between the two competing excitations. The combination of experimental and analytical theoretical tools represents a powerful playground for the design of ultrafast magneto-optical and magnetoacoustic devices. As the most strightforward application, our results can contribute to the detailed understanding of the extraordinary high acoustic transmission through the periodically microstructured surfaces and by searching for novel phenomena in the magneto-optical transmission measurements through subwavelength hole arrays patterned in hybrid metal-ferromagnet multilayers. In a broader context, the knowledge of SAW and SSLW generation in complex nanostructures can help tailor the thermal transport through interfaces.

We recently demonstrated an excitation geometry for generating magnetoelastic waves, whereby narrow-band planar elastic waves were shown to resonantly drive planar magnetization precession using Rayleigh-type surface acoustic waves. Using the transient grating geometry [6-9], we were able to demonstrate frequency tunability from 1 to $\approx 6 \mathrm{GHz}$. Here we demonstrate the broader utility of the TG technique for generating additional planar elastic waves, beyond Rayleigh SAW, which also drive magnetization precession. The TG geometry generates all elastic modes that satisfy the boundary condition imposed by the thermoelastic stress, regardless of frequency. Therefore, in our geometry we access additional elastic excitations known as surface skimming longitudinal waves. Under such excitation conditions, both elastic waves are excited simultaneously, and we show the magnetic field selective coupling of the magnetization precession to each elastic wave independently.

\section{EXPERIMENTAL TECHNIQUE}

In the TG geometry, short pulses of light at $400 \mathrm{~nm}$ are crossed onto the sample surface, which upon superposition result in a spatially periodic excitation of the sample, as shown in Fig. 1. In this excitation geometry, all elastic modes that satisfy the elastic boundary conditions are excited at the wave vector $2 \pi / \Lambda$ determined by the crossing of two beams. Subsequent to the excitation, a time-delayed $800-\mathrm{nm}$ 

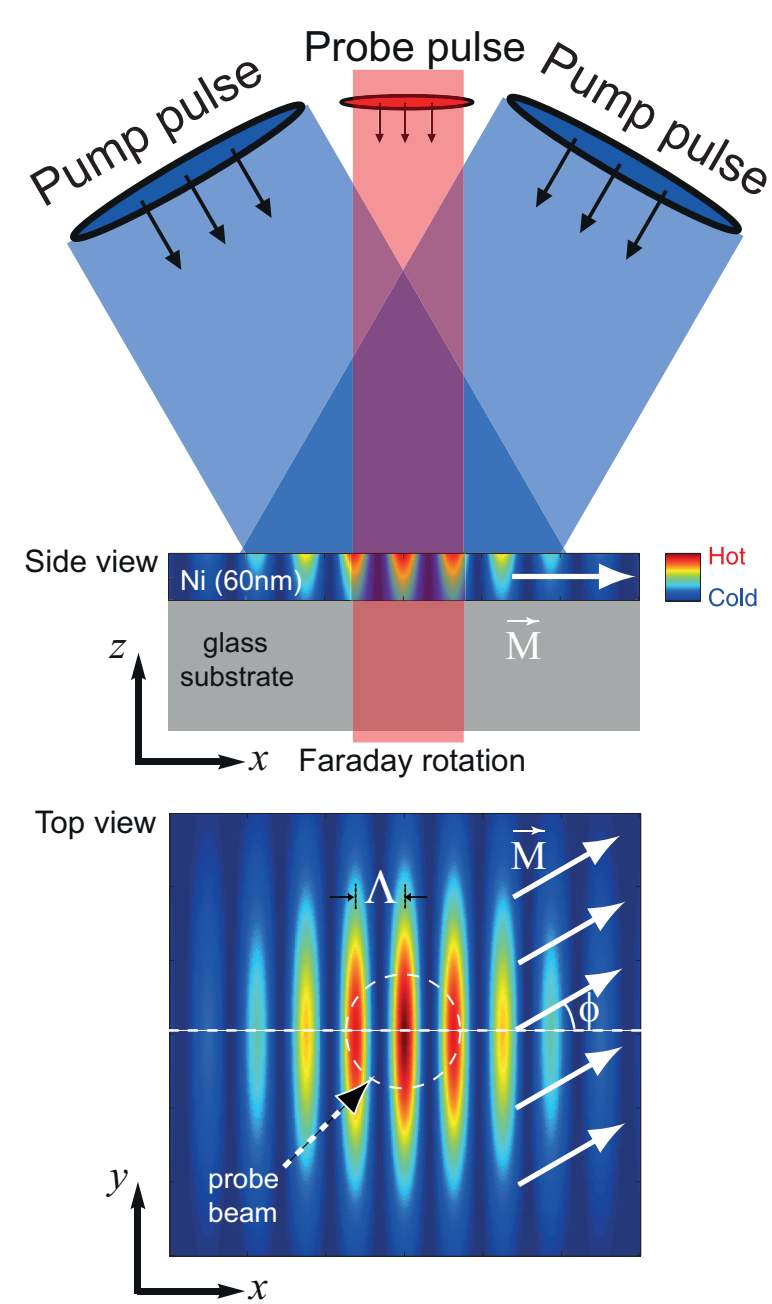

FIG. 1. Experimental geometry showing the thin nickel film with glass substrate. The transient grating is generated by two crossed femtosecond laser pump pulses, leading to a spatially periodic impulsive heating of a thin nickel film and the launching of acoustic waves along the surface of the semi-infinite glass substrate. Grating periodicities as small as $1 \mu \mathrm{m}$ can be achieved. A magnetic field can be rotated continuously in the sample plane. Faraday rotation of time-delayed optical probe pulses transmitted through the sample monitors the interaction between elastic and magnetic degrees of freedom.

probe pulse impinges normally onto the sample surface and the transmitted radiation is polarization analyzed (Faraday detection). The sample is held in a magnetic field that can be swept continuously from -1.5 to $+1.5 \mathrm{kG}$ and rotated around the sample normal.

The samples under study are composed of thin polycrystalline nickel films $(40-60 \mathrm{~nm}$, which provide qualitatively similar results) on 1-mm-thick soda lime glass (SLG) substrates. Representative data showing the grating dependence of the Faraday response are shown in Fig. 2(a) for a film thickness of $40 \mathrm{~nm}$. In contrast to our previous results [7], which were reported for the nickel/MgO sample structure, when performing measurements on glass substrates additional dynamics can be observed. Cursory evaluation of the data in Fig. 2(a) shows that both oscillation amplitude and frequency
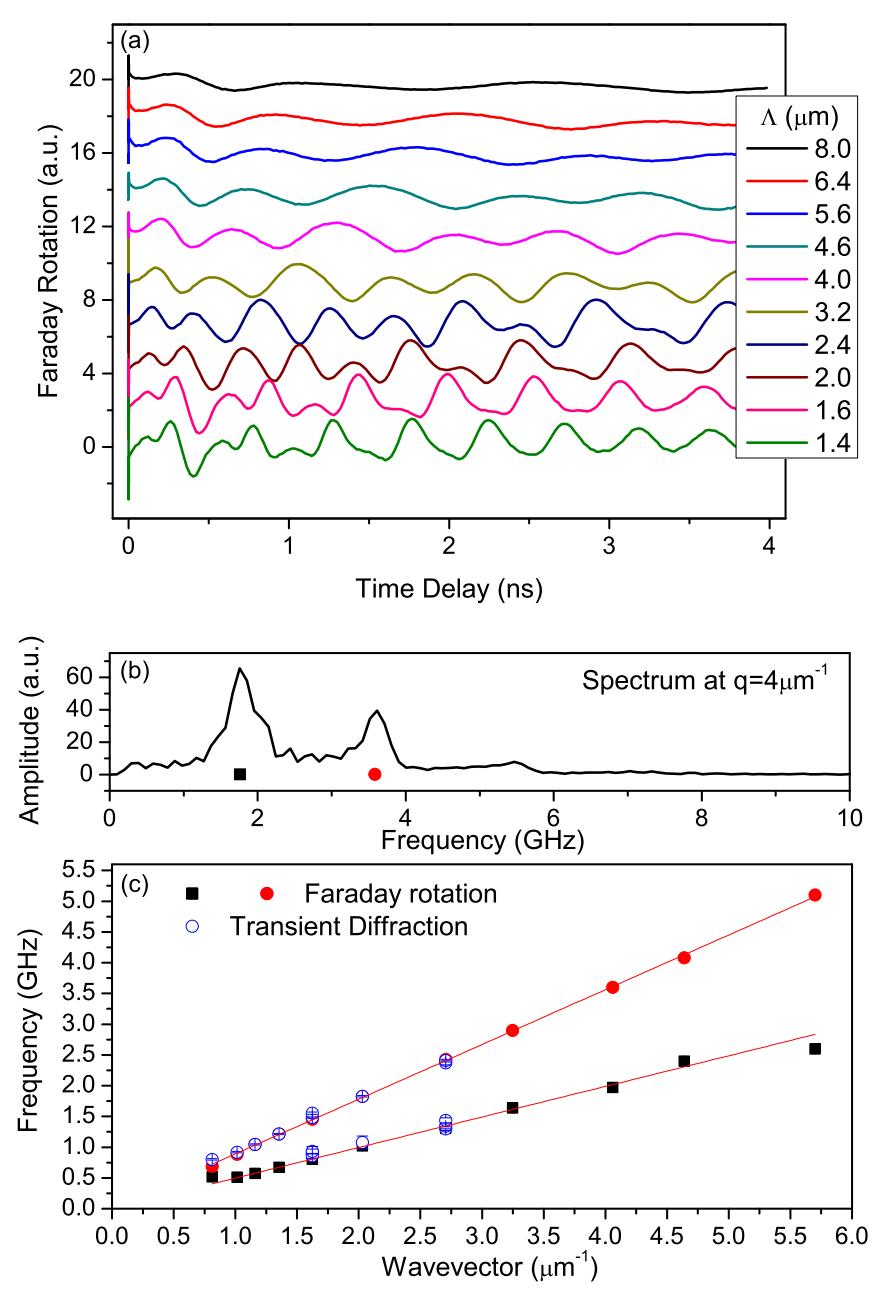

FIG. 2. (a) The time-resolved Faraday rotation measures the acoustically induced magnetization precession. As the period of the transient grating is increased, the precession decreases in frequency and amplitude. In the limit of a single pump beam excitation, the magnetic precession is completely suppressed. (c) The frequency of oscillation vs wave vector measures the velocity of acoustic propagation. Red and black data points represent frequencies extracted from (a), and blue data points are extracted from the nonmagnetic transient grating detection. Near-perfect linear dependence allows us to identify two types of excitations as the Rayleigh surface acoustic wave (lower branch) and the surface skimming longitudinal wave (upper branch) propagating at $3120 \pm 20$ and $5590 \pm 15 \mathrm{~m} / \mathrm{s}$, respectively.

reduce as the excitation grating period is increased, and eventually disappear when a single pump beam is used to excite the material (data not shown). Second, the dynamics in the first nanosecond are composed of two oscillating contributions, which suggests the presence of two distinct magnetoelastic waves.

Assignment of the modes is accomplished by plotting the frequencies of both modes as a function of excitation wave vector. In Fig. 2(b) we display the Fourier transform of the zero field response showing two oscillations. For $\Lambda=$ $1.57 \mu \mathrm{m}$, and correspondingly $k=4 \mu \mathrm{m}^{-1}$, the extracted frequencies are 1.75 and $3.6 \mathrm{GHz}$, respectively. In Fig. 2(c) these frequencies are plotted for a range of applied grating 
periodicities. Overlayed onto the Faraday response are data acquired in the conventional transient grating geometry, where light is diffracted from acoustic waves (data not shown; see Ref. [6] for details). Such an experimental scheme is known to be sensitive to the underlying elastic waves and their associated structural distortions. From the correspondence between the two detection schemes, we are able to fit a single linear relationship (with zero intercept) providing the following mode assignments and their respective velocities: The lower branch propagates at $3120 \pm 20 \mathrm{~m} / \mathrm{s}$, which we assign as the Rayleigh surface acoustic wave, the same excitation witnessed on $\mathrm{MgO}$ substrates [7]. In the long wavelength limit $(\Lambda>1 \mu \mathrm{m})$, the propagation velocity of the Rayleigh SAW in the $\mathrm{Ni} /$ substrate heterostructure is dictated by the substrate elastic constants due to the finite penetration depth $(\approx \Lambda \gg h)$ of the elastic wave. This velocity compares favorably with the Rayleigh SAW velocity of the glass substrates, $3100 \mathrm{~m} / \mathrm{s}$ for soda lime glass. The upper branch, propagating at a velocity of $5590 \pm 15 \mathrm{~m} / \mathrm{s}$, is the in-plane longitudinal acoustic wave, which has been termed previously as a surface skimming longitudinal wave (SSLW) [10], or the surface skimming bulk wave (SSBW) [11], an elastic excitation that has been used extensively for nondestructive material evaluation [12,13]. Again, the velocity is very close to the longitudinal sound velocity in glass (literature value $5400 \mathrm{~m} / \mathrm{s}$ ) due to the predominant concentration of elastic energy in the substrate.

We now discuss the effect of applying an in-plane magnetic field. As we had shown previously [7], applying a magnetic field $\vec{H}_{\text {ext }}$ in the plane of the sample provides the coupling between the elastic field at frequency $v_{a c} / \Lambda$ and the ferromagnetic resonance of the film at frequency $f_{\text {FMR }} \propto \sqrt{H_{\text {ext }}\left(H_{\text {ext }}+M\right)}$. The magnitude $H_{\text {ext }}$ of the applied field tunes the precessional FMR frequency of the film to match either SAW or SSLW frequency ( $M$ is the saturation magnetization of the thin magnetic film). The effect of having two elastic fields present provides for the selective excitation of one magnetization precession response. A representative field scan is shown in Fig. 3 where the precessional amplitude is plotted as a function of applied field (main panel) for a grating period of $1.1 \mu \mathrm{m}$ and both polarities of the applied field. The elastic frequencies at $\Lambda=1.1 \mu \mathrm{m}$ associated with SAW and SSLW are indicated by the horizontal lines over the data, while linecuts along them are scaled and displayed in the upper panel of Fig. 3. As the applied field is tuned into resonance, the oscillation amplitude of the magnetization precession peaks and then reduces as the field is tuned above resonance. For all applied fields, it should be recognized that two elastic waves are active, but the resonance condition drives a single precessional motion of the magnetization. The maxima occur at the location where the elastic driving frequency matches FMR frequency as indicated by the solid white line. Magnetization precession at low field values, where the sample may not be fully oriented, should be understood as precession occurring at positions of the sample that remain cold or unexcited by the laser source. Here, the magnitude of the magnetization is largest and the strain values are maximized.

The temporal response for three configurations of the applied magnetic field are displayed in Fig. 4. In the first two scenarios [Figs. 4(a) and 4(b)], the in-plane magnetic

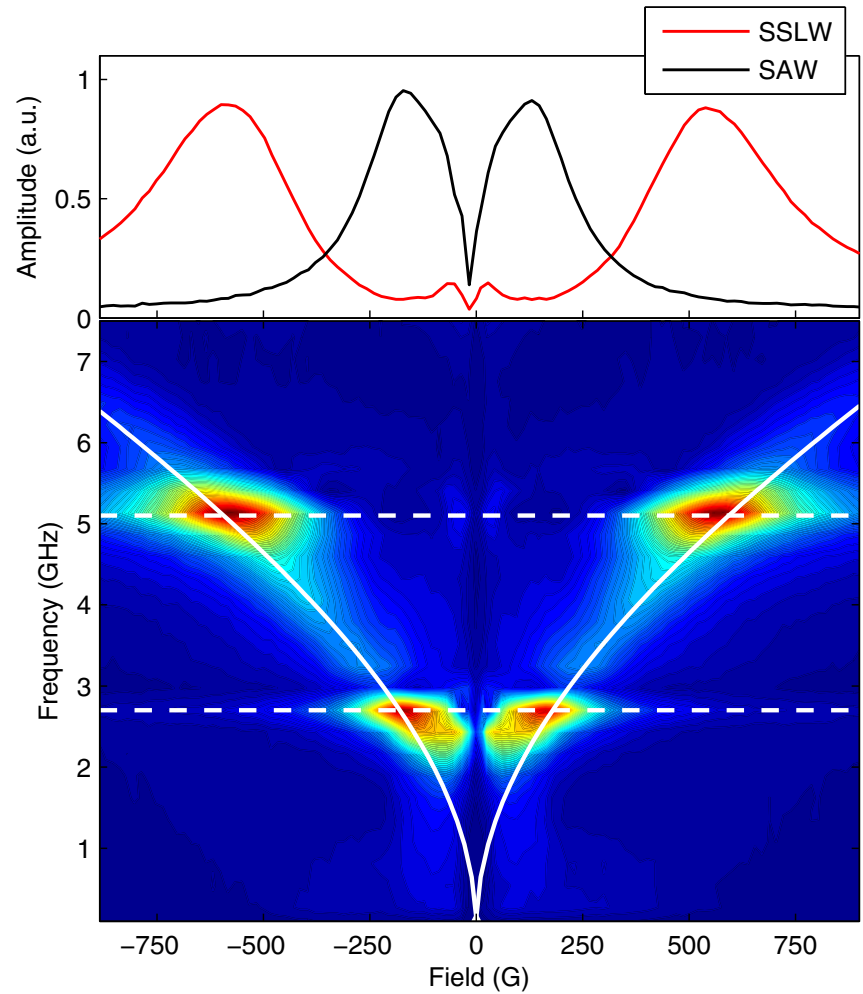

FIG. 3. Under appropriate field conditions the time-periodic effective magnetic field of the elastic wave resonantly couples to the precessional motion in the film, driving large amplitude precessional motion. For $\Lambda=1.1 \mu \mathrm{m}$, the upper frequency at $5.1 \mathrm{GHz}$ is that of the SSLW while the lower frequency at $2.8 \mathrm{GHz}$ corresponds to the SAW. At the intersection of the elastic waves (white dashed lines) and ferromagnetic resonance (white solid line) an increase in oscillation amplitude is observed. In the upper panel, scaled line cuts along the white lines are shown.

field is fixed at a small angle with respect to $\vec{k}$, while reversing the direction of the field results in the same phase of magnetization precession. The elastically generated, out-ofplane torque $[14,15]$ originates from the coupling of in-plane longitudinal strain [16], $\epsilon_{x x}$, to in-plane components, $M_{x}$ and $M_{y}$, of magnetization vector: $\partial \vec{M}_{z} / \partial t \propto \epsilon_{x x}(t) M_{x} M_{y}$. When the magnetization is inverted, i.e., $M_{x} \rightarrow-M_{x}$ and $M_{y} \rightarrow$ $-M_{y}$, the magnetoelastic torque direction and therefore the precessional direction remain unchanged. On the contrary, if the magnetization is reflected with respect the acoustic wave vector [Fig. 4(c)] $\left(M_{x} \rightarrow M_{x}\right.$ and $M_{y} \rightarrow-M_{y}$, which is equivalent to $\phi \rightarrow-\phi)$, the direction of magnetization precession changes in accordance with the above equation. We note that the foregoing discussion on magnetoelasticity is related to a myriad of other ultrafast [17-22] work as well as its connection to quasistatic straintronics $[23,24]$.

The differences in lifetime of the respective modes [Figs. 4(a) and 4(b)] can be traced directly to the nature of the elastic driving field underscoring these effects. Whereas the SAW is a surface-propagating elastic eigenwave with low energy dissipation, the SSLW is not a surface-bound wave in this strict sense, and thus significant elastic energy propagates away from the active magnetic layer. Thus the strain amplitude 

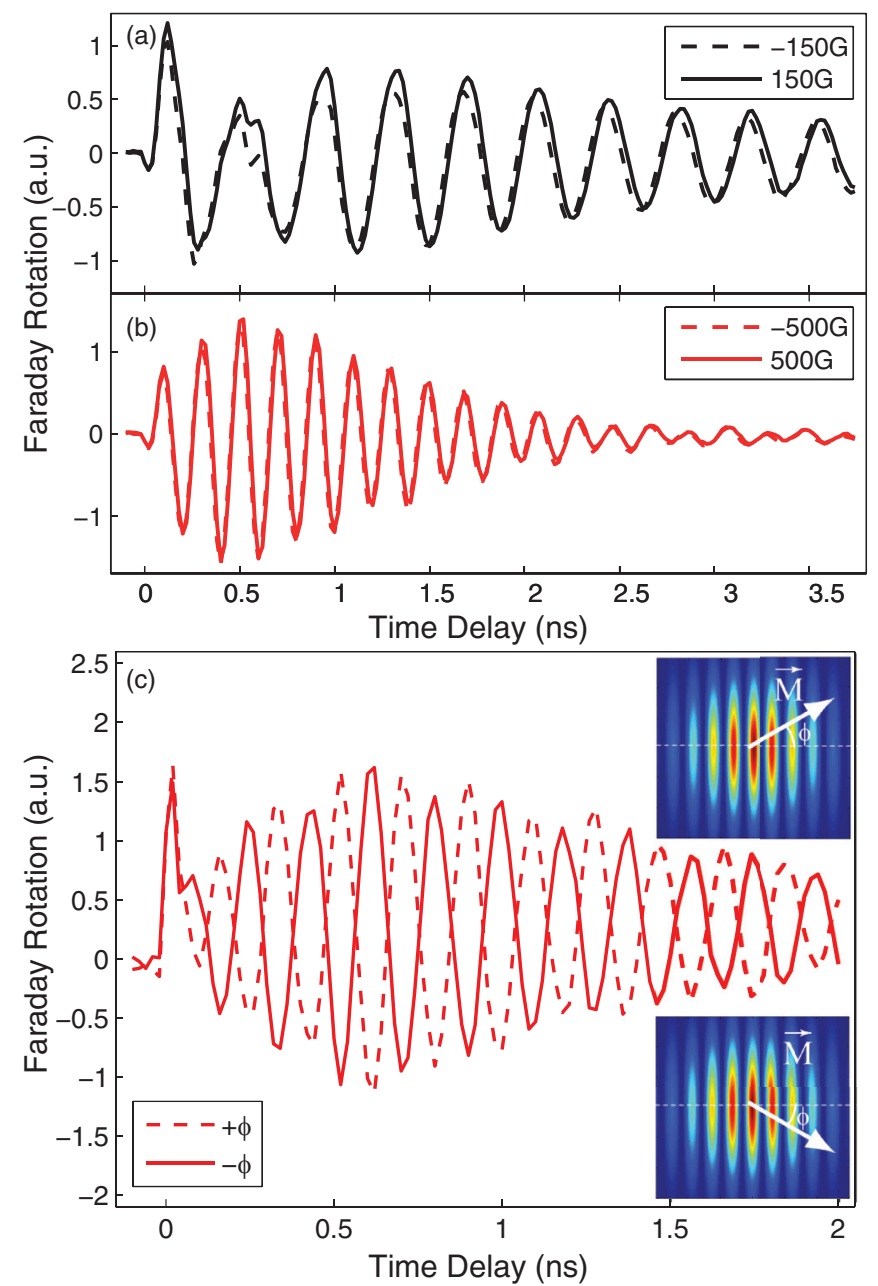

FIG. 4. Time-resolved Faraday traces at the resonances for (a) SAW and (b) SSLW, taken from Fig. 3 (main panel). Switching the polarity of the magnetic field yields nearly identical oscillation amplitudes and phases which can be understood by considering the applied torque induced by magnetoelastic coupling (see text). (c) Rotation of the applied magnetic field around the grating wave vector (geometry displayed in inset) results in an opposite sense of magnetization precession. The data shown in panel (c) is for $\Lambda=1.1 \mu \mathrm{m}$, and field tuned to the SSLW resonance.

of this higher frequency mode decays rapidly as elastic energy leaks away from the magnetically active material.

\section{GREEN'S FUNCTION CALCULATION OF TIME-DEPENDENT STRAINS}

The existence and generation of multiple elastic responses can be understood by Green's function calculations of an elastic half space loaded with a thin, elastically different, layer. Such an analytical calculation assumes absorption of pump light results in the spatially inhomogeneous distribution of temperature in the metallic layer. After the electron-phonon equilibrium is established in the film on the time scale of a few picoseconds, the thermal stress begins acting on the glass substrate.

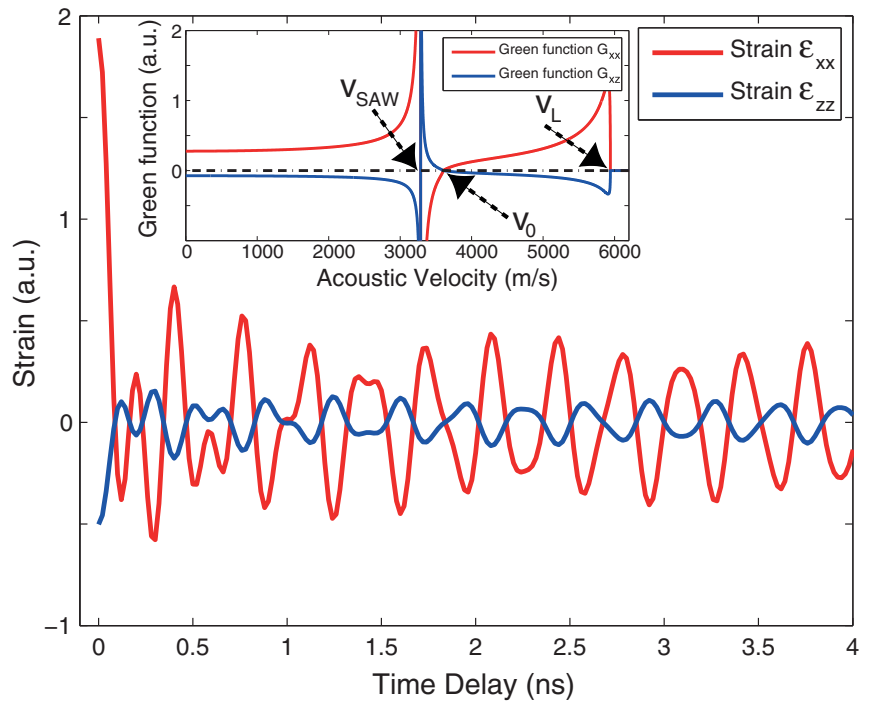

FIG. 5. Volumetric elastic strain are calculated for a bare substrate showing both in-plane and out-of-plane compressional components. The solutions to the Green's functions $G_{x x}$ and $G_{x z}$ are shown in the inset with the zero crossings indicating the velocities of respective SAW and SSLW modes. The convolution of Green's function with a periodic temperature variation (with period $\Lambda=1.1 \mu \mathrm{m}$ ) obtains the time dependence of $\epsilon_{x x}$ and $\epsilon_{z z}$ inside the glass near the surface. Calculated strains are subsequently incorporated into the magnetoelastic free energy and their effect on the magnetization drives precessional motion.

We can describe the generation of elastic transients by calculating the Green's function response in the frequency domain [25]. When an elastic half space is subjected to a temporally instantaneous, spatially localized force, the Green's functions that must be calculated are $G_{x x}$ and $G_{x z}$, which in turn can be converted into elastic displacements via a convolution with the excitation source:

$$
U_{i}(t, x)=\int G_{i x}\left(x-x^{\prime}, t\right) \cos \left(\frac{2 \pi}{\Lambda} x^{\prime}\right) d x^{\prime} .
$$

Finally strains are calculated as spatial derivatives of the displacements: $\epsilon_{i j}(t)=\frac{1}{2}\left(\frac{\partial U_{j}}{\partial i}+\frac{\partial U_{i}}{\partial j}\right)(i, j=x, z)$. Taking the source geometry into consideration and assuming both film and substrate are isotropic, displacement and gradient components along the $y$ axis equal zero: $U_{y}=0$ and $\frac{\partial U_{j}}{\partial y}=0$.

To understand the relative amplitudes of in-plane and outof-plane strains, we first show this calculation for the case of no bounding film. The Green's functions and the corresponding strains are shown in Fig. 5 with the primary result being that $\epsilon_{z z}(t)$ is smaller than the in-plane strain $\epsilon_{x x}(t)$, while both wave forms comprise signatures of both acoustic modes. In the inset of Fig. 5, the Green's function is shown and the SAW and longitudinal velocities are identified. The SSLW exhibits a broad peak extending up from the SAW velocity, whose width and amplitude determine the attenuation of the wave with propagation distance. Since the Green's function extends over a large range of velocities, the identification of individual mode contributions to the total strain is difficult. However, to some reasonable approximation, one may extract the strain contribution of the SSLW by calculating the convolution for 
all values $v>v_{0}$ (the zero-crossing point above the SAW velocity; see the inset in Fig. 5). The result takes on a form of a gradually decaying oscillation with approximately $1 / t$ amplitude dependence, as expected for acoustic diffraction in the cylindrical geometry. This procedure does not differ significantly from curve fitting of the total strain wave form, assuming two strain components which take the form of $\operatorname{SSLW}(\mathrm{t}) \sim \sin \left[v_{L} t / \Lambda\right] / t$ and $\operatorname{SAW}(\mathrm{t}) \sim \sin \left[v_{\mathrm{SAW}} t / \Lambda\right]$. Finally, when applying elastic boundary conditions we can also neglect the shear strain $\epsilon_{x z}(t)$ (which is intrinsic to SAW) since it is identically zero at the free surface.

We can now apply a similar calculation to the case of film/substrate heterostructure to study the effect of film loading on the elastic velocities, and the possible appearance of higher order elastic modes as the wave vector increases. Figure 6(a) shows the evolution of the Green's function as the thickness of nickel, $h$, is varied over a range of common experimental thicknesses: $h=20,40,60 \mathrm{~nm}$. With increasing
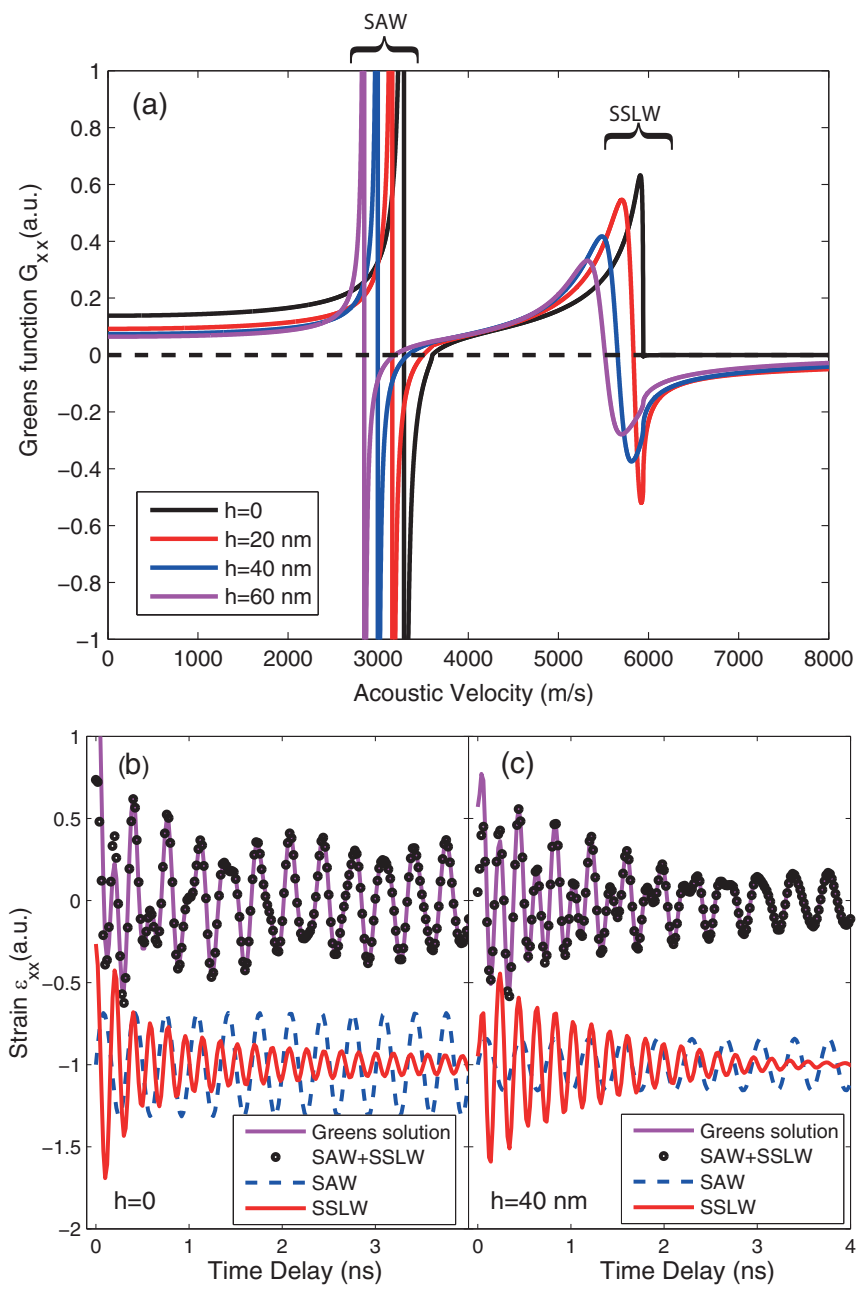

FIG. 6. The Green's function $G_{x x}$ for nickel films of different thicknesses $h=0,20,40,60 \mathrm{~nm}$ on glass substrate calculated for TG period $\Lambda=1.1 \mu \mathrm{m}$. As the film thickness increases, elastic velocities decrease while the SSLW mode also changes drastically in shape. (b, c) The time-domain signals for $h=0$ and $h=40 \mathrm{~nm}$ thin nickel on glass exhibit a decaying SSLW mode and a SAW with constant amplitude. The SSLW temporal dynamics evolve from roughly $1 / t$ dependence to exponential as the thickness increases. $h$, the velocities of both SAW and SSLW decrease, a signature of the reduced sound velocity in nickel as compared to glass. Additionally, significant modification to the Green's function shape is evident for SSLW, which in turn results in a modification to the SSLW temporal response. Performing the same procedure as detailed above, namely convolution with the Green's function for all velocities above $v_{0}$ to obtain SSLW dynamics, the resultant SSLW contribution is now well approximated by a now exponential function [compare SSLW(t) for $h=0$ and $h=40 \mathrm{~nm}$ in Figs. 6(b) and 6(c)]. The proposed approximation for SSLW(t) works very well, as confirmed in Figs. 6(b) and 6(c) by a good quantitative agreement between the exact Green's solution obtained by Eq. (1) (purple line) and the approximate solution based on mode decomposition, i.e., SAW(t) + SSLW(t) (points).

We conclude that a Green's function formulation can, at the very least, identify the underlying elastic forces which act on the magnetization through magnetoelastic coupling and verify the existence of two modes, which we identify as SAW and SSLW. Finally, the modifications to the temporal evolution of the SSLW wave guide our understanding of the TG responses and their associated magnetic modes.

A forward-looking theoretical analysis shows that as the TG periods become similar to the film thickness, $\Lambda \sim h$, the situation changes dramatically. In addition to SAW and SSLW acoustic waves, the higher order surface acoustic (Sezawa) modes emerge. In Fig. 7(a) the existence and dispersion of these higher order guided modes can be witnessed. For a fixed sample thickness, as the grating period is reduced, additional guided modes appear first as unbound, highly damped, pseudo-surface-acoustic modes and subsequently as guided modes in the film. The spatially dependent (along the film thickness) structural strain profiles likewise are modified for each mode, leading to potentially complex magnetoelastic couplings. As these higher order modes emerge, the welldefined SSLW peak becomes indistinguishable, or at the very least, highly intertwined [Fig. 7(b)] with multiple overdamped pseudo-Sezawa modes [26], while at sufficiently thin samples $(\Lambda=50 \mathrm{~nm})$, additional bound modes are clearly visible and identified as the Sezawa mode in Fig. 7(b). The analysis of this much more complex elastic mode distribution, and their respective distortion profiles, is beyond the scope of this paper. However, new techniques based on transient gratings at the FERMI@Elettra free electron laser could soon provide access to these interesting length and time scales [27] and provide experimental results to compare with calculations.

\section{COMPARISON BETWEEN GLASS AND MgO SUBSTRATES}

As a point of final discussion, we highlight the differences in measurements described here on glass substrates and those from our previous publication on $\mathrm{MgO}$ substrates [7], where only a single SAW excitation can be seen. The distinction we make between elastic waves relies on a detailed consideration of the dispersion relation, the propagation velocity, and also the elastic deformations associated with SAW and SSLW. In both TG and Faraday channels, SAW will generally exhibit oscillations over many nanoseconds of pump-probe delay time. In fact, a TG measurement clearly indicates 

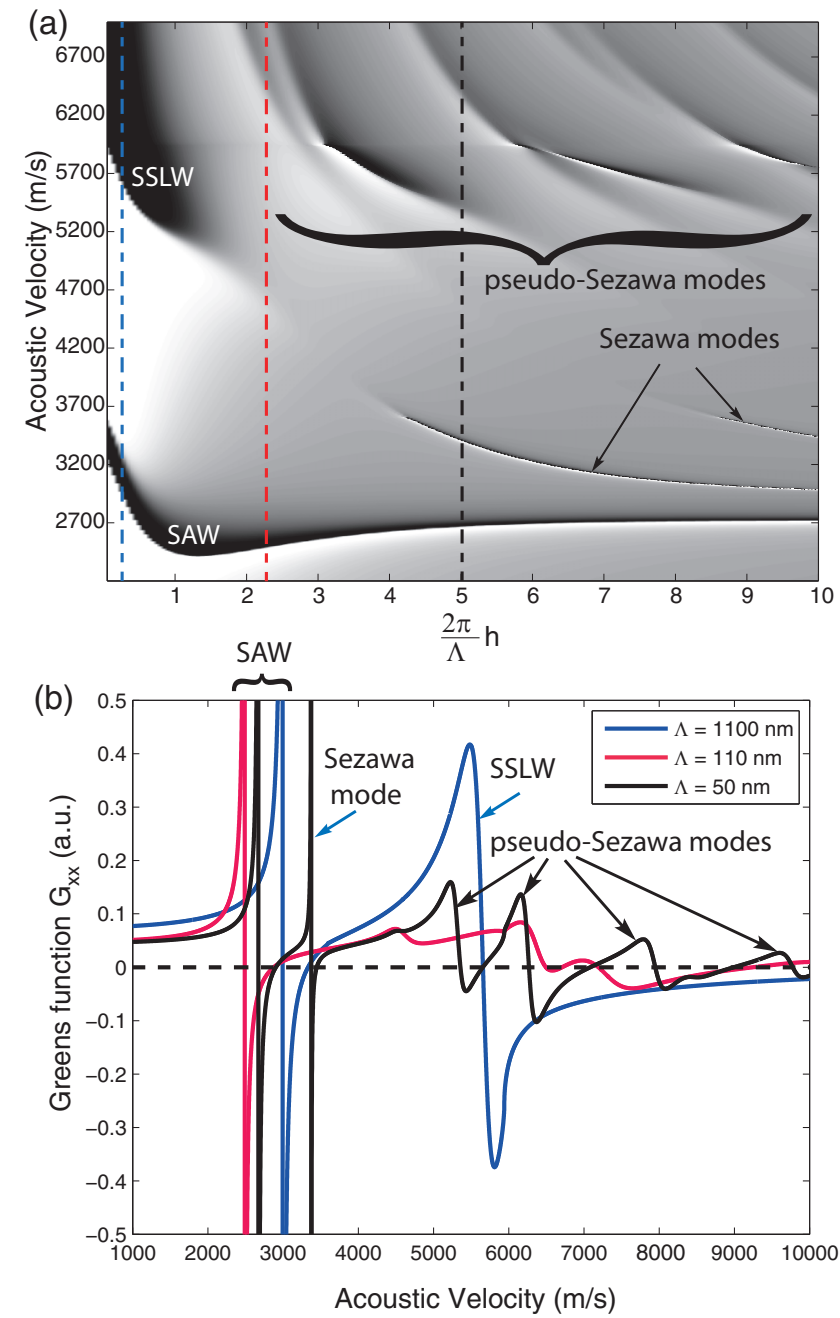

FIG. 7. The Green's function $G_{x x}$ calculated for different values $2 \pi h / \Lambda$ (upper panel). The structure of the Green's function for $\Lambda \gg$ $h$ [small abscissa, blue linecut in panel (b)] gets heavily modified for $\Lambda \sim h$, where the appearance of additional bound and unbound elastic transients appear. Vertical cross sections of the Green's function in the lower panel for a 40-nm nickel film correspond to TG periods $\Lambda=1100,110$, and $50 \mathrm{~nm}$, respectively. For $\Lambda=1100 \mathrm{~nm}$, we show the dynamics associated with two modes, SAW and SSLW, closely align with our experimental configuration. For $\Lambda=110 \mathrm{~nm}$ several overdamped (leaky) pseudo-Sezawa modes are present, while for $\Lambda=50 \mathrm{~nm}$ an additional propagating Sezawa mode at $v \sim 3400$ $\mathrm{m} / \mathrm{s}$ is observed.

that SAW oscillations persist for much longer than the time window we probe (see Fig. 2 of Janusonis et al. [7]). On the contrary, SSLW is strongly attenuated since it propagates away from the metallic surface. The elastic deformations of the surface are different for the two waves, in that SAW exhibits a strong surface deformation that is absent from SSLW, which is clearly discerned in diffraction measurements. For this reason, even though the SSLW in glass and the $\mathrm{SAW}$ in $\mathrm{MgO}$ have similar velocities and dispersions, we can clearly identify each individually. Finally, to understand the dynamics of the magnetization we also need to take into account the temperature evolution of nickel film since the amplitude of magnetization is closely linked to it. A more thorough discussion of temperature dynamics and their effect on magnetooptic sensitivity can be found here [28].

Overall, the magnetization precessional amplitudes on glass substrates are significantly larger, while the general phenomenology of resonant excitation and field tuning remains the same. The larger signal levels on the glass substrates can be accounted for by two effects, the first associated with the strain amplitudes that can be generated on the respective substrates, while the second concerns the averaging effects of the nonuniform magnetization in the Faraday detection. With regard to strain amplitudes, we note that in both materials light absorption is dictated by the bounding nickel film, and therefore the imposed temperature gradient responsible for elastic excitation is the same provided the overlayer is the same material and thickness. Since the linear expansion coefficients are similar for both substrate materials $\left(\alpha_{\mathrm{SLG}}=9 \times 10^{-6} \mathrm{~m} / \mathrm{K}, \alpha_{\mathrm{MgO}}=\right.$ $\left.9-12 \times 10^{-6} \mathrm{~m} / \mathrm{K}\right)$, the induced stress is likewise the same in both situations. However, their respective Young's moduli are markedly different, leading to a fivefold larger strain amplitude for the glass substrates. Magnetoelastic effects couple elastic strain to the magnetization direction, and thus we generally observe significantly higher signal levels in amorphous glass substrates (e.g., soda lime, fused silica, BK7, etc.), an effect that is born out in the measurements on SAW and SSLW. Second, since we monitor the average magnetization of the material, our detection scheme naturally requires a gradient in temperature along the sample surface and its associated spatially modulated magnetization amplitude. Both elastic waves in question have compressional and tensile components which act at different spatial locations at different times of the elastic wave cycle. If the magnetization were constant over the entire sample surface, then equal amplitude magnetic moments would precess out of phase, and thus result in a net zero Faraday signal. Thus the detection of average magnetization requires the precession of unequal magnetic moments. For substrates with high thermal conductivities, such as $\mathrm{MgO}$, the temperature along the sample surface quickly equilibrates due to the additional cooling into the substrate, and thus the sensitivity in the Faraday detection channel reduces. For crystalline substrates such as $\mathrm{MgO}$, the thermal conductivity is 40-50 times larger than in soda lime glass. These considerations, both with respect to strain amplitude and detection sensitivity, hold for both the SAW and SSLW signals.

\section{CONCLUSIONS}

In summary, we have demonstrated the generation of two distinct elastic waves using a single excitation geometry, based on the transient grating technique. The two modes are identified as the surface-bound Rayleigh surface acoustic wave (SAW) and the leaky surface skimming longitudinal wave (SSLW). Both elastic excitations couple to and drive the magnetization precession in a resonant fashion when an appropriate magnetic field is applied. Furthermore, since both elastic distortions are active simultaneously, we demonstrated the field-tuned selectivity of each magnetoelastic excitation. Calculations based on a Green's function approach reveal the excitation of each elastic wave via impulsive excitation and their subsequent time dynamics. Compared with the 
experimental data, we understand the temporal evolution to arise from their distinct nature at the surface of the film, namely the SSLW is not a surface-bound elastic wave while the SAW is. Our measurements are distinguished by their time-domain approach, which allowed us to witness the coupling between acoustic and magnetic degrees of freedom in real time. We envisage further experimental efforts to focus on extraordinary transmission of acoustic waves through subwavelength apertures while the magnetoelastic detection scheme provides for individual sensitivity to both elastic wave effects, opening possibilities to study competition between different elastic contributions to transmission measurements.

\section{ACKNOWLEDGMENTS}

Authors thank K. A. Nelson, A. A. Maznev, and J. Y. Duquesne for discussions. We thank M. de Roosz for assistance with e-beam sample preparation. Funding from Nouvelle équipe, nouvelle thématique et Stratégie internationale NNNTelecom de la Région Pays de La Loire, ANR-DFG "PPMINANO" (ANR-15-CE24-0032 and DFG SE2443/2), ANR "UltramoX" (ANR-14-CE26-0008), Russian Foundation for Basic Research (Grant No. 15-02-07575), and Alexander von Humboldt Stiftung is gratefully acknowledged.

The authors J.J. and C.L.C. contributed equally to this work.
[1] V. V. Temnov, Nat. Photon. 6, 728 (2012).

[2] T. Ebbesen, H. Lezec, H. Ghaemi, T. Thio, and P. Wolff, Nature (London) 391, 667 (1998).

[3] H. Lezec and T. Thio, Opt. Express 12, 3629 (2004).

[4] G. Gay, O. Alloschery, B. De Lesegno, C. O'Dwyer, J. Weiner, and H. Lezec, Nat. Phys. 2, 262 (2006).

[5] M.-H. Lu, X.-K. Liu, L. Feng, J. Li, C.-P. Huang, Y.-F. Chen, Y.-Y. Zhu, S.-N. Zhu, and N.-B. Ming, Phys. Rev. Lett. 99, 174301 (2007).

[6] J. Rogers, A. Maznev, M. Banet, and K. Nelson, Annu. Rev. Mater. Sci. 30, 117 (2000).

[7] J. Janusonis, C. L. Chang, P. H. M. van Loosdrecht, and R. I. Tobey, Appl. Phys. Lett. 106, 181601 (2015).

[8] J. D. Koralek, C. P. Weber, J. Orenstein, B. A. Bernevig, S.-C. Zhang, S. Mack, and D. D. Awschalom, Nature (London) 458, 610 (2009).

[9] R. I. Tobey, M. E. Siemens, M. M. Murnane, H. C. Kapteyn, D. H. Torchinsky, and K. A. Nelson, Appl. Phys. Lett. 89, 091108 (2006).

[10] B. Xu, Z. Shen, X. Ni, and J. Lu, J. Appl. Phys. 95, 2116 (2004).

[11] M. Lewis, Ultrasonics Symposium, 1977 (IEEE, New York, 1977), pp. 744-752.

[12] S. Sathish, R. Martin, and T. Moran, J. Acoust. Soc. Am. 115, 165 (2004).

[13] A. Abbas, Y. Guillet, J. M. Rampnoux, P. Rigail, E. Mottay, B. Audoin, and S. Dilhaire, Opt. Express 22, 7831 (2014).

[14] O. Kovalenko, T. Pezeril, and V. V. Temnov, Phys. Rev. Lett. 110, 266602 (2013).

[15] L. Dreher, M. Weiler, M. Pernpeintner, H. Huebl, R. Gross, M. S. Brandt, and S. T. B. Goennenwein, Phys. Rev. B 86, 134415 (2012).

[16] In our previous manuscript, we assigned $\epsilon_{x z}$ as the leading strain component responsible for the observed dynamics for the external magnetic field being parallel to TG wave vector. Upon further angle-dependent measurements, we can now definitively identify the majority of dynamics being driven by $\epsilon_{x x}$ for $\phi>1$ deg.

[17] A. V. Scherbakov, A. S. Salasyuk, A. V. Akimov, X. Liu, M. Bombeck, C. Brueggemann, D. R. Yakovlev, V. F. Sapega, J. K. Furdyna, and M. Bayer, Phys. Rev. Lett. 105, 117204 (2010).

[18] T. L. Linnik, A. V. Scherbakov, D. R. Yakovlev, X. Liu, J. K. Furdyna, and M. Bayer, Phys. Rev. B 84, 214432 (2011).

[19] M. Bombeck, J. V. Jaeger, A. V. Scherbakov, T. Linnik, D. R. Yakovlev, X. Liu, J. K. Furdyna, A. V. Akimov, and M. Bayer, Phys. Rev. B 87, 060302 (2013).

[20] J.-W. Kim, M. Vomir, and J.-Y. Bigot, Phys. Rev. Lett. 109, 166601 (2012)

[21] J. V. Jaeger, A. V. Scherbakov, T. L. Linnik, D. R. Yakovlev, M. Wang, P. Wadley, V. Holy, S. A. Cavill, A. V. Akimov, A. W. Rushforth et al., Appl. Phys. Lett. 103, 032409 (2013).

[22] J. V. Jaeger, A. V. Scherbakov, B. A. Glavin, A. S. Salasyuk, R. P. Campion, A. W. Rushforth, D. R. Yakovlev, A. V. Akimov, and M. Bayer, Phys. Rev. B 92, 020404 (2015).

[23] K. Roy, S. Bandyopadhyay, and J. Atulasimha, Appl. Phys. Lett. 99, 063108 (2011).

[24] L. Thevenard, J. Y. Duquesne, E. Peronne, H. J. von Bardeleben, H. Jaffres, S. Ruttala, J.-M. George, A. Lemaitre, and C. Gourdon, Phys. Rev. B 87, 144402 (2013).

[25] A. Maznev and A. Every, Int. J. Eng. Sci. 35, 321 (1997).

[26] A. Every, Meas. Sci. Technol. 13, R21 (2002).

[27] F. Bencivenga, R. Cucini, F. Capotondi, A. Battistoni, R. Mincigrucci, E. Giangrisostomi, A. Gessini, M. Manfredda, I. P. Nikolov, E. Pedersoli et al., Nature (London) 520, 205 (2015).

[28] J. Janušonis, T. Jansma, C. L. Chang, Q. Liu, A. Gatilova, A. M. Lomonosov, V. Shalagatskyi, T. Pezeril, V. V. Temnov, and R. I. Tobey, Sci. Rep. 6, 29143 (2016). 